\title{
Senotherapeutics: emerging strategy for healthy aging and age-related disease
}

\author{
Eok-Cheon Kim \& Jae-Ryong Kim ${ }^{*}$ \\ Department of Biochemistry and Molecular Biology, Smart-aging Convergence Research Center, College of Medicine, Yeungnam \\ University, Daegu 42415, Korea
}

Cellular senescence (CS) is one of hallmarks of aging and accumulation of senescent cells (SCs) with age contributes to tissue or organismal aging, as well as the pathophysiologies of diverse age-related diseases (ARDs). Genetic ablation of SCs in tissues lengthened health span and reduced the risk of age-related pathologies in a mouse model, suggesting a direct link between SCs, longevity, and ARDs. Therefore, senotherapeutics, medicines targeting SCs, might be an emerging strategy for the extension of health span, and prevention or treatment of ARDs. Senotherapeutics are classified as senolytics which kills SCs selectively; senomorphics which modulate functions and morphology of SCs to those of young cells, or delays the progression of young cells to SCs in tissues; and immune-system mediators of the clearance of SCs. Some senolytics and senomorphics have been proven to markedly prevent or treat ARDs in animal models. This review will present the current status of the development of senotherapeutics, in relation to aging itself and ARDs. Finally, future directions and opportunities for senotherapeutics use will discussed. This knowledge will provide information that can be used to develop novel senotherapeutics for health span and ARDs. [BMB Reports 2019; 52(1): 47-55]

\section{INTRODUCTION}

Cellular senescence (CS) is initially defined as an irreversible growth arrest of normal somatic cells, and has been proposed to contribute to tissue and organismal aging itself, and to be an intrinsic safeguard against tumor progression (1). SCs have been shown to have diverse phenotypes, including cellular flattening and hypertrophy, senescence-associated $\beta$-galactosidase activity $(\mathrm{SA} \beta \mathrm{G})$, senescence-associated secretory phenotype

*Corresponding author. Tel: +82-53-640-6931; Fax: +82-53-6297093; E-mail: kimjr@ynu.ac.kr

https://doi.org/10.5483/BMBRep.2019.52.1.293

Received 19 October 2018

Keywords: Age-related disease, Aging, Cellular senescence, Health span, Senotherapeutics
(SASP), resistance to apoptotic cell death, alterations in nuclear structure and senescence-associated heterochromatic foci (SAHF), mitochondrial expansion, and signaling events, including upregulation of cell cycle inhibitors and -pro-survival effectors (2). SCs accumulated in various tissues in mice with age, and comprised $5-40 \%$ of the total cells, depending on the tissue types (3). Although their number are relatively small in tissues, SCs can cause extensive dysfunction of the microenvironment and damage to surrounding cells and tissues, due to their pro-inflammatory senescence-associated secretory phenotype (SASP). Accumulating evidence suggests that CS plays an important role in diverse biological processes, such as embryonic development, diabetes, host immunity, wound healing, tissue renewal, as well as fibrosis, cardiovascular diseases, and cancer (4).

Since aging is one of major risk factors of human diseases, a variety of aging interventions have been developed to extend health span and to prevent or treat ARDs in in vitro and in vivo experimental models. Caloric restriction (CR) is the only intervention shown to increase health span as well as to decrease the risk of ARDs in nonhuman primates (5). Recently, clinical trials of CR in non-obese humans revealed that a $15 \%$ lower calorie intake for 2 years delayed metabolism accompanied by reduced oxidative damage, suggesting that CR could also slow down the aging process in humans (6). Although CR can enhance healthy aging, the inconvenience of most subjects to maintain $C R$ for a longtime limits its application. Therefore, caloric restriction mimetics (7), and calorie restriction diets or fasting-mimicking diets (8) have been proposed as alternatives. Elucidation of the mechanisms by which aging is regulated also suggested a variety of compounds and medicines, including sirtuin activators (9), AMP dependent protein kinase (AMPK) activators (10), mammalian target of rapamycin (mTOR) inhibitors (11), autophagy activators (12), that might be applicable for use in aging intervention. In addition, the use of geroprotectors, compounds and medicines that slow down aging, and thus lengthen the lifespan of model organisms has also been proposed (13). In present, a curated database of geroprotectors is available, and includes 259 compounds in 13 animal models from yeast to human, obtained from 2,408 literature (http://geroprotectors.org). An old story tells the rejuvenation

ISSN: 1976-670X (electronic edition)

Copyright (c) 2019 by the The Korean Society for Biochemistry and Molecular Biology

(ㄷ) This is an open-access article distributed under the terms of the Creative Commons Attribution Non-Commercial License (http://creativecommons.org/licenses/by-nc/4.0) which permits unrestricted non-commercial use, distribution, and reproduction in any medium, provided the original work is properly cited. 
effects of young blood. Heterochronic parabiosis, in which an aged mouse and a young one were joined surgically, revealed that some factors in young blood, such as growth differentiation factor 11 with controversial reports and oxytocin enhanced tissue regeneration, and led to improvement of aging phenotypes (14). Similarly, transfusion of young serum also retarded age-related impairments in cognitive function and synaptic plasticity in aged mice $(15,16)$.

Although CS is one of hallmarks of aging (17), and accumulation of SCs with age has been suggested to be associated with aging and ARDs (18), direct evidence of a causal relationship between CS and aging or ARDs has only recently been validated in rodent models. Furthermore, senotherapeutics, have been implicated as novel strategies for aging intervention in applications designed to extend healthy aging and to prevent or treat ARDs.

\section{DIRECT LINKAGE OF CS TO AGING AND ARDs}

Baker et al., reported the first direct evidence of a direct causal relationship of CS to ARDs in 2011 (19). For the clearance of senescent cells in mice, a transgenic strategy, was employed, using INK-ATTAC derived from $p 16^{\operatorname{lnk} 4 a}$, a well-known marker of CS, in which senescent cells were selectively eliminated by apoptotic cell death upon administration of AP20187. The INK-ATTAC transgenic mice were bred onto a $B u b R 1^{H / H}$ progeroid mouse background to obtain $B u b R 1^{H / H} ;$;NK-ATTAC mice. The authors demonstrated that the animals treated with
AP20187 from early (weaning time) or late (5 months) in life, had reduced numbers of $p 16^{\operatorname{lnk} 4 a}$-positive senescent cells, and progression of $p 16^{\operatorname{lnk} 4 a}$-mediated age-related phenotypes in adipose tissue and muscle was delayed (19). Five years later, Baker et al., reported more concrete evidence of the direct linkage of CS to aging itself and ARDs (20). This time, they demonstrated effects of the clearance of $p 16^{\ln k 4 a}$-positive senescent cells in both male and female INK-ATTAC transgenic mice of two distinct genetic backgrounds (C57BL/6 and mixed). AP20187 treatment from 12 months to 18 months increased the median lifespan of both C57BL/6 and mixed background mice by $24 \%$, and prolonged the heath span in C57BL 6 mice by $18 \%$, and by $25 \%$ in mixed background mice. In addition, they demonstrated that AP20187 attenuated age-related functional and structural deterioration of multiple organs, without any detrimental side effects to adipose tissue, kidney, or heart (20). Genetic ablation of senescent cells, using the INK-ATTAC transgenic mice further revealed that clearance of $p 16^{\text {Ink4a }}$-positive senescent cells improved age-related lipodystrophy (21), hepatic steatosis (22), age-related cardiac function and bone loss (23), and tau-mediated cerebral pathologies (24). Studies on another $p 16^{\operatorname{lnk} 4 a}$-based transgenic mouse, named the $p 16^{\operatorname{lnk} 4 a}$-trimodality reporter (p16-3MR) mouse, in which $p 16^{\operatorname{lnk} 4 a}$-positive senescent cells were cleared by treatment with ganciclovir, revealed that removal of senescent macrophages attenuated atherosclerotic plaque formation in $L D L R^{-1-}$ background mice (25), and osteoarthritis (26). In addition to genetic clearance of senescent cells,

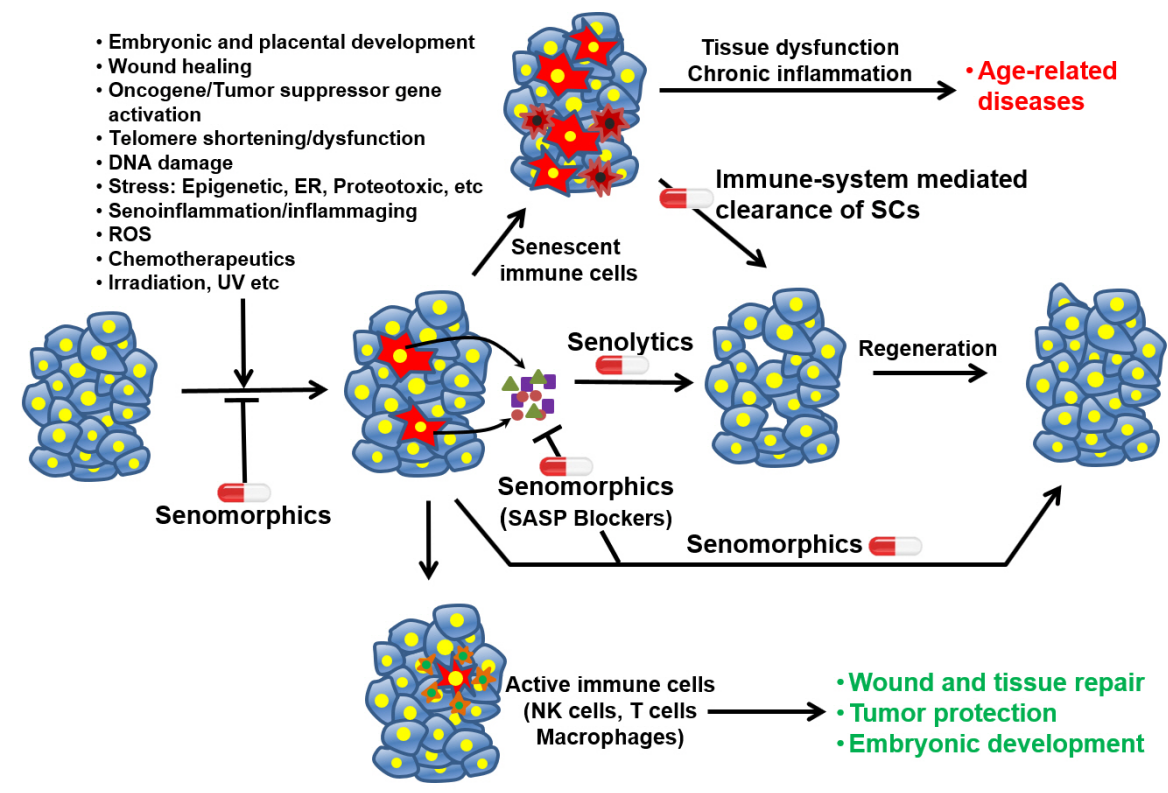

Senotherapeutics

Non-senescent cell
SASP
४ Active immune cell
Senescent immune cell
Senescent cell
Fig. 1. Senotherapeutics targeting SCs. CS induced by diverse factors is involved in many biological processes, embryonic development, tissue homeostasis, and tissue dysfunction, thus contributing to age-related pathologies and lifespan. Therefore, senotherapeutics targeting SCs is an emerging strategy of aging intervention for extension of health span and prevention and treatment of ARDs. Senotherapeutics is comprised of 3 classes: senolytics which kill SCs selectively; senomorphics which modulates or even reverses the phenotypes of SCs to those of young cells by interfering with triggers of CS, targeting SCs directly, or blocking SASP: and mediators of the immune-system clearance of SCs. 
transplanting senescent ear fibroblasts into the knee region induced osteoarthritis in mice (27). Transplanting relatively small numbers of senescent cells into young mice was reportedly sufficient to cause persistent physical dysfunction, as well as to spread cellular senescence to host tissues, which led to reduced survival (28). Therefore, these proof-of-principle experiments revealed the direct linkage of CS to aging and ARDs, and suggest that therapeutic interventions to remove senescent cells or block their effects might represent a novel strategy to lengthen health span and prevent or treat ARDs in human (20).

\section{SENOLYTICS}

Since elimination of SCs using genetic approaches mitigated aging and ARDs, pharmacological intervention targeting SCs, named as senotherapeutics, has been proposed. Senotherapeutics are classified as senolytics, which selectively kill SCs; senomorphics which modulate SCs by blocking SASP; and senoinflammation, the immune system-mediated clearance of

\section{SCs (Fig. 1).}

In 2015, Zhu et al., reported the first senolytics, dasatinib, a protein tyrosine kinase inhibitor, and quercetin, a plant flavonoid (29). Based on transcriptomic analysis, they discovered that SCs increased the expression of pro-survival networks, which led to resistance to apoptosis. The authors screened 46 candidate drugs for their ability to eliminate SCs in vitro and found that dasatinib was effective against senescent human preadipocytes, and that quercetin was effective against senescent human endothelial cells and mouse bone marrow-derived mesenchymal stem cells (BM-MSCs). Finally, they showed that combination of dasatinib and quercetin reduced SC burden in chronologically aged, radiation-exposed, and $\operatorname{Ercc}^{-/ 4}$-progeroid mice, which resulted in extension of health span and reduction of age-related pathologies (29). Their seminal demonstration on the feasibility of selectively ablating senescent cells by pharmacological intervention and the efficacy of senolytics for alleviating symptoms of frailty and extending health span in mice, prompted development of other senolytics.

Table 1. Senolytic candidates

\begin{tabular}{|c|c|c|c|c|}
\hline Agents & Target/Pathway & Effective senescent cells & Outcomes in vivo & Ref. \\
\hline Dasatinib + Quercetin & $\begin{array}{l}\text { Pan-receptor tyrosine } \\
\text { kinase/Multiple pathways }\end{array}$ & $\begin{array}{l}\text { HUVECs } \\
\text { Preadipocytes } \\
\text { MEFs } \\
\text { BM-MSCs }\end{array}$ & $\begin{array}{l}\downarrow \text { Atherosclerosis } \\
\downarrow \text { Osteoporosis } \\
\downarrow \text { Hepatic steatosis } \\
\downarrow \text { Pulmonary fibrosis } \\
\uparrow \text { Exercise capacity } \\
\uparrow \text { Vasomotor function } \\
\uparrow \text { Cardiac function } \\
\uparrow \text { Lifespan }\end{array}$ & $\begin{array}{l}22 \\
23 \\
28 \\
29 \\
31 \\
32\end{array}$ \\
\hline ABT-263 & $\begin{array}{l}\text { BCl-2 family (BCl-2, } \\
\text { BCL-XL, BCl-W) }\end{array}$ & $\begin{array}{l}\text { HUVECS } \\
\text { IMR90 } \\
\text { MEFs } \\
\text { Wl-38 }\end{array}$ & $\begin{array}{l}\uparrow \text { Hematopoietic and muscle stem cell function } \\
\downarrow \text { Atherosclerotic lesion formation }\end{array}$ & $\begin{array}{l}33 \\
34\end{array}$ \\
\hline ABT-737 & $\begin{array}{l}\text { Bcl-2 family (Bcl-2, Bcl-xL, } \\
\text { Bcl-W) }\end{array}$ & IMR90 & $\begin{array}{l}\uparrow \text { Hair follicle stem cell function } \\
\downarrow \text { IR-induced lung injury }\end{array}$ & 35 \\
\hline $\begin{array}{l}\text { A1331852 } \\
\text { A1155463 }\end{array}$ & Bcl-2 family (Bcl-xL) & $\begin{array}{l}\text { HUVECs } \\
\text { IMR90 } \\
\text { Cholangiocytes }\end{array}$ & $\downarrow$ Liver fibrosis & 37 \\
\hline 17-AAG Geldanamycin & HSP90 & $\begin{array}{l}\text { MEFs } \\
\text { MSCs } \\
\text { IMR90 } \\
\text { HUVECs } \\
\text { Wl-38 }\end{array}$ & $\begin{array}{l}\downarrow \text { Age-related symptoms } \\
\uparrow \text { Health span }\end{array}$ & 38 \\
\hline Fisetin & PI3K/AKT & HUVECS & Not reported & 36 \\
\hline Piperlongumine & Multiple pathways & WI-38 & Not reported & 39 \\
\hline Quercetin-3-D-galactose & Multiple pathways & HCAECs & Not reported & 40 \\
\hline UBX0101 & MDM2/p53 & Chondrocytes & $\downarrow$ Osteoarthritis & 26 \\
\hline Panobinostat & HDAC & $\begin{array}{l}\text { NSCLC cell lines } \\
\text { HNSCC cell lines }\end{array}$ & Not reported & 41 \\
\hline FOXO4-DRI peptide & p53/p21/serpine & IMR90 & $\begin{array}{l}\downarrow \text { Liver toxicity induced by doxorubicin } \\
\downarrow \text { Frailty } \\
\uparrow \text { Hair growth } \\
\uparrow \text { Renal function }\end{array}$ & 44 \\
\hline
\end{tabular}


Until now, seven classes of senolytics have been reported, including kinase inhibitors, a Bcl-2 family inhibitors, natural compounds, a p53 binding inhibitor, heat shock protein 90 (HSP90) inhibitors, UBX0101, and a histone deacetylase (HDAC) inhibitor (30) (Table 1). The combined administration of dasatinib and quercetin improved the pathologies of diverse ARDs, including cardiac aging (29), atherosclerosis (31), osteoporosis (23), pulmonary fibrosis (32), hepatic steatosis (22), and Alzheimer's disease (24) by clearing SCs in the tissues. Recently, the combination of dasatinib and quercetin was reported to enhance physical function and to lengthen health span and lifespan in old mice (28).

Resistance of SCs to apoptotic cell death, due to upregulation of $\mathrm{Bcl}-2$ and $\mathrm{Bcl}-\mathrm{xL}$ suggested inhibitors of these anti-apoptotic proteins as useful candidates for senolytics. In this regard, ABT-263, ABT-737, A1331852, and A1155463 were reported as senolytic candidates in in vitro, as well as in in vivo models. ABT-263, which binds to the inhibitory domain of anti-apoptotic Bcl-2, Bcl-xL, and Bcl-W, effectively cleared SCs, senescent bone marrow hematopoietic stem cells (HSCs), and senescent muscle stem cells (MuSCs) from either irradiated or normally aged mice, and led to mitigation or rejuvenation of HSCs and MuSCs in both animal models (33). ABT-263 selectively decreased viability of some senescent cells, such as senescent human umbilical vein epithelial cells (HUVECs), IMR90 human lung fibroblasts, and murine embryonic fibroblasts (MEFs), but not human primary preadipocytes (34). ABT-263 eliminated senescent foam cell macrophages in atherosclerotic lesions, consequently blocking progression of atherosclerosis in $L D L R^{-1-}$ mice (25). ABT-737 selectively killed senescent IMR90 cells induced by etoposide, $H$-Ras activation, and replication in vitro (35). ABT-737 treatment also efficiently cleared senescent lung epithelial cells of irradiated mice, and senescent epidermal cells of $p 14^{A R F}$ transgenic mice, and resulted in an increase in hair follicle stem cell proliferation (35). A1331852 and A1155463, selective $\mathrm{BCl}-\mathrm{xL}$ inhibitors, induced apoptosis of irradiation-induced senescent HUVECs and IMR90 cells, but not of preadipocytes (36). A1331852 reduced liver fibrosis through depletion of senescent cholangiocytes and reduction of serum levels of SASP factors in primary sclerosing cholangitis model of multidrug-resistance 2 knockout mice (37).

Inhibitors of HSP90, 17-N-allylamino-17-demethoxygeldanamycin (17-AAG) and geldanamycin, were suggested as a new class of senolytic candidates from the screening of 97 autophagy regulators in oxidative stress-induced senescent ErCC $^{-1-}$ mouse embryonic fibroblasts (MEFs) (38). These HSP90 inhibitors induced apoptosis of senescent MEFs, murine mesenchymal stem cells (MSCs), IMR-90, HUVECs, and WI-38 cells, induced by oxidative stress, irradiation, or replication, in vitro. They selectively eliminated $p 16^{\operatorname{lnk} 4 a}$-positive cells, and thereby delayed the onset of several age-related symptoms, and ultimately lengthened the health span of ErCC $^{-/ 4}$-progeroid mice (38).
In addition to quercetin, natural phytochemicals, piperlongumine $(\mathrm{PL})$ and fisetin, were reported to have senolytic activities in in vitro experiments. PL preferentially induced apoptosis in human Wl-38 fibroblasts senesced by ionizing radiation, replicative exhaustion, or ectopic expression of the oncogene Ras, through an ROS-independent pathway (39). Fisetin selectively killed IR-induced senescent HUVECs, but not senescent IMR90 or pre-adipocytes (36). Quercetin3-D-galactose (Q3G) has been suggested to be a potential senolytic agent, that was reported to kill senescent endothelial cells, but not HUVECs (40).

UBX0101, a small molecule inhibitor of the MDM2/p53 protein interaction, was revealed to be a potent senolytic candidate (26). Intra-articular injection of UBX0101 eliminated SCs accumulated in the articular cartilage and synovium, and resulted in attenuation of the development of post-traumatic osteoarthritis (OA) in aged mice (26). UBX0101 also cleared SCs by inducing apoptosis and improved the cartilage-forming ability of chondrocytes from human OA tissue (26). UBX0101 is the first senolytic under Phase 1 clinical trial in patients with OA of the knee (https://clinicaltrials.gov/ct2/show/NCT03513016).

In addition to primary cells, senolytics targeting cancer cells have been also proposed. Panobinostat, an FDA approved HDAC inhibitor, was identified to have senolytic activity in chemotherapy-induced senescence of cancer cells, non-small cell lung cancer, and head and neck squamous cell carcinoma cell lines (41). During standard chemotherapy, senescenceassociated events, such as decreased histone acetylation, enhancement of Bcl-2 family proteins, and SASP production occurred in cancer cells $(42,43)$. The latter events are a biomarker of senescence and a target of senotherapeutics. Chemotherapy-induced senescence has reported to be involved in the increased invasion of cancer cells, due to epithelial-to-mesenchymal transition in thyroid cancer (42), as well as acquisition of stem-cell-related properties in B-cell lymphoma (43). Thus, senolytics targeting senescent cancer cells might be a new strategy for treatment cancer metastasis and cancer stemness.

Forkhead box protein O4 (FOXO4) was identified as a pivotal protein involved in the viability of SCs. Using bioinformatic analysis of RNA sequence data from IR-induced senescent IMR90 cells, FOXO4-D-retro-inverso (FOXO4-DRI) peptide was demonstrated to be a new class of senolytic candidates (44). FOXO4-DRI peptide blocked the interaction of FOXO4 and p53 in senescent cells, which induced apoptosis of senescent IMR90 cells and HUVECs. Administration of the peptide improved fitness, hair density, and kidney function in both $X p d^{T T D / T T D}$-progeroid and naturally aged mice (44).

Based on the countable lists of senolytics candidates, research on senolytics is still in its infancy, but growing rapidly. Since most senolytics target known proteins, such as p53, a tyrosine kinase, $\mathrm{HSP} 90, \mathrm{Bcl}-2$, and $\mathrm{Bcl}-\mathrm{xL}$, elucidation of novel targets specific to CS is necessary for further 
development of senolytics. In addition, senolytics demonstrated cell-specific effects in killing SCs, suggesting CS might be differentially regulated depending on cell or tissue-specific factors. Further unbiased exploration of senolytics using chemical libraries or drug repositioning might contribute to the development of novel senolytics, as well as to the elucidation of novel mechanisms of CS.

\section{SENOMORPHICS}

Senomorphics is a wide range of agents that can modulate the phenotypes of SCs to those of young cells through interfering with senoinflammation/inflammaging, senescence-related signal pathways, and SASP, without induction of SC apoptosis (Table 2). Senomorphics include previous anti-aging or anti-senescence compounds, such as telomerase activator (45), CRMs (7), caloric restriction diets (8), sirtuin activators (46), mTOR inhibitors (47), antioxidants (48), anti-inflammatory agents targeting senoinflammation or inflammaging (49), autophagy activators (12), and proteasome activators (50). This review will not cover the aforementioned anti-aging or anti-senescence agents as senomophics. Instead, we have included recent results on agents exhibiting senomorphic activity, based on selective markers of cellular senescence, such as SASP and SABG in in vitro and in vivo experimental models.

Senoinflammation or inflammaging refers to chronic, sterile, low-grade, and unresolved inflammation characterized upon aging $(51,52)$. Nuclear factor-kappa B (NF- $\mathrm{kB})$ activated in aging and ARDs, is a key transcription factor mediating senoinflammation or inflammaging and its activation has been linked to known regulators of the aging process, such as insulin/insulin-like growth factor (IGF)-1, FOXO, SIRT, mTOR and DNA damage (53). Therefore, inhibition of NF-KB has been suggested as a potential target of senomorphics. A peptide inhibitor of IKK, the NF-KB-activating kinase, reduced cellular senescence in vitro and in vivo, and delayed the age-related symptoms and pathologies of $\mathrm{ErCC}^{-/ 4}$-progeroid mice (54). In addition, SASP in SCs also plays an important role in senoinflammation or inflammaging. Thus, blocking of SASP in SCs might be a plausible target of senomorphics. SASP is regulated by the Janus kinase (JAK)/STAT pathway, and pharmacological inhibitors of JAK in SCs suppressed senoinflammation, alleviated age-related tissue dysfunction, and enhanced physical function in old mice (55).

Ataxia-telangiectasia mutated (ATM) kinase, a serine/threonine protein kinase activated by DNA double strand breaks, functions in the regulation of CS (2). Therefore, ATM kinase inhibitor, KU-60019, was identified as a potential senomorphic candidate. KU-60019 induced the functional recovery of the lysosome/autophagy system, coupled with mitochondrial functional recovery and metabolic reprogramming (56).

Progerin, a truncated form of lamin A protein involved in Hutchitson-Gilford progeria syndrome (HGPS), accumulated with progressive telomere shortening during $\mathrm{CS}$ of normal human fibroblasts, and suggested a causative role in CS (57). A

Table 2. Senomorphic candidates

\begin{tabular}{|c|c|c|c|}
\hline Agents & Target pathway & Effects & Ref. \\
\hline NBD peptide & IKK/NFB pathway & $\begin{array}{l}\text { Delaying aging symptoms and chronic diseases in } \\
\text { ErcC1 }^{-1} \text {-progeroid mice }\end{array}$ & 54 \\
\hline JAK inhibitor (ruxolitinib) & JAK (Janus kinase) pathway & Alleviation of the SASP and frailty in old mice & 55 \\
\hline KU-60019 & ATM kinase & $\begin{array}{l}\text { Functional recovery of senescent fibroblasts through } \\
\text { lysosomal-mitochondrial axis } \\
\text { Acceleration of cutaneous wound healing in aged mice }\end{array}$ & 56 \\
\hline $\mathrm{JH} 4$ & Progerin/lamin AVC & $\begin{array}{l}\text { Alleviation of nuclear deformation and reduction of senescence } \\
\text { markers in HGPS and aged cells } \\
\text { Mitigation of age-related pathologies and extension of lifespan } \\
\text { in the HGPS-progeroid mice }\end{array}$ & 58 \\
\hline Juglanin & Not reported & $\begin{array}{l}\text { Inhibition of doxorubicin-induced senescence of HDFs and } \\
\text { HUVECs and replicative cellular senescence of HDFs }\end{array}$ & 59 \\
\hline Quercetin-3-O- $\beta$-D-glucuronide & Not reported & $\begin{array}{l}\text { Inhibition of doxorubicin-induced and replicative senescence of } \\
\text { HDFs and HUVECs }\end{array}$ & 60 \\
\hline$(-)$-Loliolide & Not reported & $\begin{array}{l}\text { Inhibition of doxorubicin-induced and replicative senescence of } \\
\text { HDFs }\end{array}$ & 61 \\
\hline Quercetagetin 3,4'-dimethyl ether & Not reported & $\begin{array}{l}\text { Inhibition of doxorubicin-induced and replicative senescence of } \\
\text { HUVECs }\end{array}$ & 62 \\
\hline ESC-CM & PDGF/FGF pathway & $\begin{array}{l}\text { Decreases in senescence phenotypes of HDFs and HUVECs } \\
\text { Acceleration of the skin wound healing in mice }\end{array}$ & 63 \\
\hline Mmu-miR-291a-3p & TGFBR2/p21 pathway & $\begin{array}{l}\text { Inhibition of senescence phenotypes of HDFs and HUVECs } \\
\text { Acceleration of the excisional skin wound healing in aged mice }\end{array}$ & 64 \\
\hline
\end{tabular}


small molecule, $\mathrm{JH} 4$, interfering binding of progerin and lamin $\mathrm{A} / \mathrm{C}$, has been shown to be a senomorphic candidate. $\mathrm{JH} 4$ alleviated nuclear deformation and reversed senescence markers, such as growth arrest and SABG activity, in HGPS and aged cells. Administration of $\mathrm{JH} 4$ markedly mitigated several age-related pathologies and extended lifespan in HGPS-progeroid mice (58).

Natural compounds derived from plants, including juglanin (59), quercetin-3-O- $\beta$-D-glucuronide (60), (-)-loliolide (61), and quercetagetin 3,4'-dimethyl ether (62) were identified as senomorphic candiates, when they decreased SA $\beta$ G and p53 levels in senescent HDFs and HUVECs.

Embryonic stem cells (ESCs) and ESC conditioned medium (CM) have been suggested as a source of anti-aging interventions that modulate the phenotypes of SCs. ESC-CM and ESC-CM derived factors, such as growth factor and miRNAs, reportedly might also be senomorphic candidates. ESC-CM markedly improved the phenotypes of SCs in HDFs and HUVECs, through a PDGF-FGF mediated pathway and accelerated wound healing in an in vivo mouse model (63). In addition, mmu-miR-291a-3p, enclosed in exosomes secreted from ESCs, was found to mitigate the phenotypes of SCs in HDFs and HUVECs, through a transforming growth factor beta receptor 2 (TGFBR2)-p21 pathway, and accelerated wound healing in aged mice (64).

\section{IMMUNE-SYSTEM MEDIATED CLEARANCE OF SCs}

SCs in tissues can activate both innate and adaptive immune responses, and are eliminated by the immune system to maintain tissue homeostasis mainly, through the DNA damage response (65). Clearance of SCs contributed to the regulation of tissue homeostasis, such as wound healing (66), hepatic fibrosis (67), and embryonic/placenta development (68). However, inefficient clearance of SCs due to aging of immune system or senescence immunosurveillence was shown to facilitate their accumulation in various tissues with age, and led to aging and ARDs (65). SCs revealed upregulation of MHC class I polypeptide-related sequence A (MICA) and UL16 binding protein 2 (ULBP2), ligands of an activating natural killer (NK) cell receptor (NKG2D), which induced NK-mediated clearance of SCs and decreased liver fibrosis (69). Dipeptidyl peptidase 4 (DPP4) was selectively upregulated in the cell membrane of senescent fibroblasts, but not in proliferating cells or DPP4-positive SCs preferentially eliminated by antibody-mediated NK cell-mediated cytotoxicity, and suggested a possible immunotherapeutic strategy for targeting SCs (70) (Fig. 1). In addition to NK cells, $\mathrm{CD} 4^{+} \mathrm{T}$ cells and macrophages were also involved in clearance of SCs, and prevented tumor development (71) and their contribution to embryonic development (68). Since NKG2D ligands are also expressed in many cancer cells, strategies to improve immunosurveillance already being developed as cancer therapy, might be repurposed to target SCs for the alleviation of aging and ARDs $(18,72,73)$ (Table 3). T cells engineered to express the NKG2D chimeric antigen receptor (CAR), which recognizes NKG2D ligands on the surface of SCs, may be used to target SCs (65). CD9 is upregulated in the cell membrane of SCs (74), and CD9 antibody conjugated nanoparticles or liposomes preferentially delivered drugs to SCs and inhibited phenotypes of SCs in HDFs $(75,76)$. Therefore, antibody-mediated targeted drug delivery to SCs represents another immunotherapeutic strategy against SCs. Although some cell surface proteins preferentially upregulated in SCs have been shown to be involved in immune-system mediated clearance of SCs, more cell membrane proteins specific to diverse SCs should be investigated. Their identification would contribute to the development of senescence vaccines, targeting senescencespecific antigens, and promote development of novel strategies for immune-system mediated clearance of SCs.

\section{CONCLUSION}

Recent accumulating evidence has suggested a causal linkage between CS, and aging and ARDs, and has led to the development of senotherapeutics targeting SCs. These senotherapeutics are classified as senolytics, senomorphics, and immune-system mediators of the clearance of SCs, and represent an emerging strategy of aging intervention for healthy aging, and prevention and treatment of ARDs. Similar to chemotherapeutics, the development of novel senotherapeutics must be more active and promote aging research on the mechanisms of CS regulation to identify novel targets. However, additional crucial issues must be overcome before senotherapeutics are applied in clinical practice. Although preclinical animal experiments revealed no significant side

Table 3. Immune-system mediated clearance of SCs

\begin{tabular}{lll}
\hline Surface proteins & Expression & Therapeutic cells \\
\hline MICA, ULBP2 & $\begin{array}{c}\text { Replicative, oncogene-induced, and DNA } \\
\text { damage-induced senescent fibroblasts } \\
\text { Senescent fibroblasts }\end{array}$ & Clearance of SCs by NK cells \\
DPP4 & $\begin{array}{l}\text { Replicative and doxorubicin-induced senescent } \\
\text { HUVECs and HDFs }\end{array}$ & $\begin{array}{c}\text { Clearance of SCs by NK cells } \\
\text { Delivery of senomorphics by CD9-antibody } \\
\text { cD9 }\end{array}$ \\
\hline
\end{tabular}


effects of some senolytics, further in-depth and careful analyses on potential adverse effects of long-term administration of senotherapeutics is essential for its successful application in healthy aging and ARDs of humans. Occasionally, imagination becomes realty. In 1997, GATTACA, a science fiction (SF) film directed by Andrew Niccol, highlighted a eugenics issue in genetically modified babies in a not-too-distant future society. In 2010, he released other SF film, IN TIME, in which people are genetically engineered to stop aging on their $25^{\text {th }}$ birthday in 2169. What do you think of the two SF films? Are they still fantasy? In the future, senotherapeutics will be available in markets and contribute to healthy aging and prevention and treatment of ARDs.

\section{ACKNOWLEDGEMENTS}

This research was supported by Medical Research Center Program (2015R1A5A2009124) and Basic Science Research Program (218C000399), through the National Research Foundation of Korea (NRF) funded by the Ministry of Science and ICT.

\section{CONFLICTS OF INTEREST}

The authors have no conflicting interests.

\section{REFERENCES}

1. Hayflick L and Moorhead PS (1961) The serial cultivation of human diploid cell strains. Exp Cell Res 25, 585-621

2. Campisi J (2013) Aging, cellular senescence, and cancer. Annu Rev Physiol 75, 685-705

3. Wang C, Jurk D, Maddick M, Nelson G, Martin-Ruiz C and von Zglinicki T (2009) DNA damage response and cellular senescence in tissues of aging mice. Aging Cell 8, 311-323

4. He S and Sharpless NE (2017) Senescence in Health and Disease. Cell 169, 1000-1011

5. Leong I (2018) Sustained caloric restriction in health. Nat Rev Endocrinol 14, 322

6. Redman LM, Smith SR, Burton JH, Martin CK, II'yasova D and Ravussin E (2018) Metabolic Slowing and Reduced Oxidative Damage with Sustained Caloric Restriction Support the Rate of Living and Oxidative Damage Theories of Aging. Cell Metab 27, 805-815 e804

7. Roth GS and Ingram DK (2016) Manipulation of health span and function by dietary caloric restriction mimetics. Ann N Y Acad Sci 1363, 5-10

8. Wei M, Brandhorst S, Shelehchi $M$ et al (2017) Fasting-mimicking diet and markers/risk factors for aging diabetes, cancer, and cardiovascular disease. Sci Transl Med 9, 377

9. Mitchell SJ, Martin-Montalvo A, Mercken EM et al (2014) The SIRT1 activator SRT1720 extends lifespan and improves health of mice fed a standard diet. Cell Rep 6, 836-843

10. Burkewitz K, Zhang Y and Mair WB (2014) AMPK at the nexus of energetics and aging. Cell Metab 20, 10-25

11. Harrison DE, Strong R, Sharp ZD et al (2009) Rapamycin fed late in life extends lifespan in genetically heterogeneous mice. Nature 460, 392-395

12. Nakamura $S$ and Yoshimori $T$ (2018) Autophagy and Longevity. Mol Cells 41, 65-72

13. Moskalev A, Chernyagina E, Kudryavtseva A and Shaposhnikov M (2017) Geroprotectors: A Unified Concept and Screening Approaches. Aging Dis 8, 354-363

14. Conese M, Carbone A, Beccia E and Angiolillo A (2017) The Fountain of Youth: A Tale of Parabiosis, Stem Cells, and Rejuvenation. Open Med (Wars) 12, 376-383

15. Villeda SA, Plambeck KE, Middeldorp J et al (2014) Young blood reverses age-related impairments in cognitive function and synaptic plasticity in mice. Nat Med 20, 659-663

16. Castellano JM, Mosher KI, Abbey RJ et al (2017) Human umbilical cord plasma proteins revitalize hippocampal function in aged mice. Nature 544, 488-492

17. Lopez-Otin C, Blasco MA, Partridge L, Serrano $M$ and Kroemer G (2013) The hallmarks of aging. Cell 153, 1194-1217

18. Munoz-Espin D and Serrano M (2014) Cellular senescence: from physiology to pathology. Nat Rev Mol Cell Biol 15, 482-496

19. Baker DJ, Wijshake T, Tchkonia T et al (2011) Clearance of p16lnk4a-positive senescent cells delays ageingassociated disorders. Nature 479, 232-236

20. Baker DJ, Childs BG, Durik M et al (2016) Naturally occurring p16(Ink4a)-positive cells shorten healthy lifespan. Nature 530, 184-189

21. Xu M, Palmer AK, Ding $\mathrm{H}$ et al (2015) Targeting senescent cells enhances adipogenesis and metabolic function in old age. Elife 4, e12997

22. Ogrodnik M, Miwa S, Tchkonia T et al (2017) Cellular senescence drives age-dependent hepatic steatosis. Nat Commun 8, 15691

23. Farr JN, Xu M, Weivoda MM et al (2017) Targeting cellular senescence prevents age-related bone loss in mice. Nat Med 23, 1072-1079

24. Bussian TJ, Aziz A, Meyer CF, Swenson BL, van Deursen JM and Baker DJ (2018) Clearance of senescent glial cells prevents tau-dependent pathology and cognitive decline. Nature 562, 578-582

25. Childs BG, Baker DJ, Wijshake T, Conover CA, Campisi J and van Deursen JM (2016) Senescent intimal foam cells are deleterious at all stages of atherosclerosis. Science 354, 472-477

26. Jeon $\mathrm{OH}$, Kim C, Laberge RM et al (2017) Local clearance of senescent cells attenuates the development of post-traumatic osteoarthritis and creates a pro-regenerative environment. Nat Med 23, 775-781

27. Xu M, Bradley EW, Weivoda MM et al (2017) Transplanted Senescent Cells Induce an OsteoarthritisLike Condition in Mice. J Gerontol A Biol Sci Med Sci 72, 780-785

28. Xu M, Pirtskhalava T, Farr JN et al (2018) Senolytics improve physical function and increase lifespan in old age. Nat Med 24, 1246-1256 
29. Zhu Y, Tchkonia T, Pirtskhalava T et al (2015) The Achilles' heel of senescent cells: from transcriptome to senolytic drugs. Aging Cell 14, 644-658

30. Niedernhofer LJ and Robbins PD (2018) Senotherapeutics for healthy ageing. Nat Rev Drug Discov 17, 377

31. Roos CM, Zhang B, Palmer AK et al (2016) Chronic senolytic treatment alleviates established vasomotor dysfunction in aged or atherosclerotic mice. Aging Cell 15, 973-977

32. Schafer MJ, White TA, lijima K et al (2017) Cellular senescence mediates fibrotic pulmonary disease. Nat Commun 8, 14532

33. Chang J, Wang Y, Shao L et al (2016) Clearance of senescent cells by ABT263 rejuvenates aged hematopoietic stem cells in mice. Nat Med 22, 78-83

34. Zhu Y, Tchkonia T, Fuhrmann-Stroissnigg $\mathrm{H}$ et al (2016) Identification of a novel senolytic agent, navitoclax, targeting the Bcl-2 family of anti-apoptotic factors. Aging Cell 15, 428-435

35. Yosef R, Pilpel N, Tokarsky-Amiel R et al (2016) Directed elimination of senescent cells by inhibition of BCL-W and BCL-XL. Nat Commun 7, 11190

36. Zhu Y, Doornebal EJ, Pirtskhalava T et al (2017) New agents that target senescent cells: the flavone, fisetin, and the BCL-XL inhibitors, A1331852 and A1155463. Aging (Albany NY) 9, 955-963

37. Moncsek A, Al-Suraih MS, Trussoni CE et al (2018) Targeting senescent cholangiocytes and activated fibroblasts with B-cell lymphoma-extra large inhibitors ameliorates fibrosis in multidrug resistance 2 gene knockout (Mdr2(-/-) ) mice. Hepatology 67, 247-259

38. Fuhrmann-Stroissnigg H, Ling YY, Zhao J et al (2017) Identification of HSP90 inhibitors as a novel class of senolytics. Nat Commun 8, 422

39. Wang Y, Chang J, Liu X et al (2016) Discovery of piperlongumine as a potential novel lead for the development of senolytic agents. Aging (Albany NY) 8, 2915-2926

40. Hwang HV, Tran DT, Rebuffatti MN, Li CS and Knowlton AA (2018) Investigation of quercetin and hyperoside as senolytics in adult human endothelial cells. PLoS One 13, e0190374

41. Samaraweera L, Adomako A, Rodriguez-Gabin A and McDaid HM (2017) A Novel Indication for Panobinostat as a Senolytic Drug in NSCLC and HNSCC. Sci Rep 7, 1900

42. Kim YH, Choi YW, Lee J, Soh EY, Kim JH and Park TJ (2017) Senescent tumor cells lead the collective invasion in thyroid cancer. Nat Commun 8, 15208

43. Milanovic M, Fan DNY, Belenki D et al (2018) Senescenceassociated reprogramming promotes cancer stemness. Nature 553, 96-100

44. Baar MP, Brandt RMC, Putavet DA et al (2017) Targeted Apoptosis of Senescent Cells Restores Tissue Homeostasis in Response to Chemotoxicity and Aging. Cell 169, 132-147.e116

45. Liu P, Zhao H and Luo Y (2017) Anti-Aging Implications of Astragalus Membranaceus (Huangqi): A Well-Known Chinese Tonic. Aging Dis 8, 868-886

46. Hubbard BP and Sinclair DA (2014) Small molecule
SIRT1 activators for the treatment of aging and age-related diseases. Trends Pharmacol Sci 35, 146-154

47. Lamming DW, Ye L, Sabatini DM and Baur JA (2013) Rapalogs and mTOR inhibitors as anti-aging therapeutics. J Clin Invest 123, 980-989

48. Si H and Liu D (2014) Dietary antiaging phytochemicals and mechanisms associated with prolonged survival. J Nutr Biochem 25, 581-591

49. Soto-Gamez A and Demaria M (2017) Therapeutic interventions for aging: the case of cellular senescence. Drug Discov Today 22, 786-795

50. Chondrogianni N, Voutetakis K, Kapetanou M et al (2015) Proteasome activation: An innovative promising approach for delaying aging and retarding age-related diseases. Ageing Res Rev 23, 37-55

51. Chung HY, Lee EK, Choi YJ et al (2011) Molecular inflammation as an underlying mechanism of the aging process and age-related diseases. J Dent Res 90, 830-840

52. Franceschi C, Garagnani P, Vitale G, Capri M and Salvioli S (2017) Inflammaging and 'Garb-aging'. Trends Endocrinol Metab 28, 199-212

53. Tilstra JS, Clauson CL, Niedernhofer LJ and Robbins PD (2011) NF-kappaB in Aging and Disease. Aging Dis 2, 449-465

54. Tilstra JS, Robinson AR, Wang J et al (2012) NF-kappaB inhibition delays DNA damage-induced senescence and aging in mice. J Clin Invest 122, 2601-2612

55. Xu M, Tchkonia T, Ding $\mathrm{H}$ et al (2015) JAK inhibition alleviates the cellular senescence-associated secretory phenotype and frailty in old age. Proc Natl Acad Sci U S A 112, E6301-6310

56. Kang HT, Park JT, Choi K et al (2017) Chemical screening identifies ATM as a target for alleviating senescence. Nat Chem Biol 13, 616-623

57. Cao K, Blair CD, Faddah DA et al (2011) Progerin and telomere dysfunction collaborate to trigger cellular senescence in normal human fibroblasts. J Clin Invest $121,2833-2844$

58. Lee SJ, Jung YS, Yoon MH et al (2016) Interruption of progerin-lamin $\mathrm{A} / \mathrm{C}$ binding ameliorates Hutchinson-Gilford progeria syndrome phenotype. J Clin Invest 126, 3879-3893

59. Yang HH, Hwangbo K, Zheng MS et al (2014) Inhibitory effects of juglanin on cellular senescence in human dermal fibroblasts. J Nat Med 68, 473-480

60. Yang $\mathrm{HH}$, Hwangbo $\mathrm{K}$, Zheng MS et al (2014) Quercetin-3-O-beta-D-glucuronide isolated from Polygonum aviculare inhibits cellular senescence in human primary cells. Arch Pharm Res 37, 1219-1233

61. Yang HH, Hwangbo K, Zheng MS et al (2015) Inhibitory effects of (-)-loliolide on cellular senescence in human dermal fibroblasts. Arch Pharm Res 38, 876-884

62. Yang HH, Zhang H, Son JK and Kim JR (2015) Inhibitory effects of quercetagetin 3,4'-dimethyl ether purified from Inula japonica on cellular senescence in human umbilical vein endothelial cells. Arch Pharm Res 38, 1857-1864

63. Bae YU, Choi JH, Nagy A, Sung HK and Kim JR (2016) Antisenescence effect of mouse embryonic stem cell conditioned medium through a PDGF/FGF pathway. FASEB J 30, 1276-1286 
64. Bae YU, Son Y, Kim CH et al (2018) Embryonic stem cell-derived mmu-miR-291a-3p inhibits cellular senescence in human dermal fibroblasts through the TGF-receptor 2 pathway. J Gerontol A Biol Sci Med Sci [Epub ahead of print]

65. Burton DGA and Stolzing A (2018) Cellular senescence: Immunosurveillance and future immunotherapy. Ageing Res Rev 43, 17-25

66. Demaria M, Ohtani N, Youssef SA et al (2014) An essential role for senescent cells in optimal wound healing through secretion of PDGF-AA. Dev Cell 31, 722-733

67. Krizhanovsky V, Yon M, Dickins RA et al (2008) Senescence of activated stellate cells limits liver fibrosis. Cell 134, 657-667

68. Munoz-Espin D, Canamero M, Maraver A et al (2013) Programmed cell senescence during mammalian embryonic development. Cell 155, 1104-1118

69. Sagiv A, Burton DG, Moshayev Z et al (2016) NKG2D ligands mediate immunosurveillance of senescent cells. Aging (Albany NY) 8, 328-344

70. Kim KM, Noh JH, Bodogai M et al (2017) Identification of senescent cell surface targetable protein DPP4. Genes
Dev 31, 1529-1534

71. Kang TW, Yevsa T, Woller N et al (2011) Senescence surveillance of pre-malignant hepatocytes limits liver cancer development. Nature 479, 547-551

72. van Deursen JM (2014) The role of senescent cells in ageing. Nature 509, 439-446

73. Childs BG, Durik M, Baker DJ and van Deursen JM (2015) Cellular senescence in aging and age-related disease: from mechanisms to therapy. Nat Med 21, 1424-1435

74. Kim TW, Kim HJ, Lee C et al (2008) Identification of replicative senescence-associated genes in human umbilical vein endothelial cells by an annealing control primer system. Exp Gerontol 43, 286-295

75. Thapa RK, Nguyen HT, Jeong JH et al (2017) Progressive slowdown/prevention of cellular senescence by CD9targeted delivery of rapamycin using lactose-wrapped calcium carbonate nanoparticles. Sci Rep 7, 43299

76. Nguyen HT, Thapa RK, Shin BS et al (2017) CD9 monoclonal antibody-conjugated PEGylated liposomes for targeted delivery of rapamycin in the treatment of cellular senescence. Nanotechnology 28, 095101 